\title{
EVALUATION OF LEARNING CURVE AND PERIPHERAL AWARENESS USING A NOVEL MULTIRESOLUTION FOVEATED LAPAROSCOPE
}

\author{
Marissa Lovett \\ David Biffar \\ Allan Hamilton
Arizona Simulation Technology Education Center
University of Arizona
1501 N. Campbell Avenue
Tucson, AZ, USA
Lovett1@email.arizona.edu
\{dbiffar,allan\}@surgery.arizona.edu

\author{
Jeremy Katz \\ Sangyoon Lee \\ Hong Hua
}

\author{
Mike Nguyen \\ USC Institute of Urology \\ University of Southern California \\ 1441 Eastlake Avenue \\ Los Angeles, CA, USA \\ Mike.Nguyen@med.usc.edu
}

\begin{abstract}
Laparoscopic surgery relies on effective visualization of areas of interest and surrounding tissue. The limited field of view of traditional laparoscopes increases the risk of peripheral tissue injury. To address this limitation, a multiresolution foveated laparoscope (MRFL) was designed to enhance peripheral awareness while maintaining focus on the area of interest. Peripheral awareness and learner effect were evaluated with novices $(n=13)$ and experts $(n=8)$. These subjects completed a modified Fundamentals of Laparoscopy Surgery task using a traditional laparoscope, MRFL with autotracking (AT), and MRFL without autotracking (NoAT). Subjects using the traditional laparoscope performed better than MRFL AT and MRFL NoAT in task completion time and collision number. The learner effect was steeper with use of MRFL. In multiple tested parameters, MRFL NoAT performed as well as the traditional laparoscope while providing the added benefits of enhanced field of view and multiple viewing perspectives. These findings warrant further development of MRFL.
\end{abstract}

Keywords: Laparoscope, Learning Curve, Peripheral Awareness, Tracking, Multiresolution Foveated Laparoscope 


\section{INTRODUCTION}

Minimally invasive surgery using laparoscopy has become a standard approach to decrease surgery related infection and recovery time (Hata et al. 2014, Jaschinski et al. 2018, Wu et al. 2017). Laparoscopic surgery relies on effective visualization of an area of interest and surrounding tissue using a handheld camera and novice assistant. The limited field of view with traditional laparoscopes is a major challenge to visualization. With the laparoscope focused on an area of interest, there is increased risk of tissue injury upon insertion and removal of tools in the periphery. Tracking of these tools adds additional time to the surgery and risks losing the optimal plane of focus. Manipulation of the camera to the area of interest is also complicated by communication with a novice camera holder. Seeking to address these challenges, authors Hua, Katz, and Lee at the University of Arizona College of Optical Sciences built a novel multiresolution foveated laparoscope (MRFL) shown in Figure 1. Our previous evaluations of the MRFL demonstrate a wider field of view, larger optimal working distance, a two image display to allow simultaneous viewing of both zoomed and wide view perspectives, and autotracking to allow the zoomed view to automatically follow the tip of the instrument to the field of interest (Hua and Liu 2018; Qin and Hua 2016a; Qin and Hua 2016b; Qin et al. 2013; Qin et al. 2014). The wide field of view and larger working distance allow the MRFL to capture a larger surgical area $(160 \mathrm{~mm} \times 120 \mathrm{~mm})$ compared to the traditional laparoscope (56 $\mathrm{mm}$ x $42 \mathrm{~mm}$ ) (Qin et al. 2014). The multiple perspectives simultaneously displayed with the MRFL provide a larger field of view than the single view display used by traditional laparoscopes. The MRFL autotracking system provides a method to maneuver the zoomed view without the camera holder required for traditional laparoscopy. Studies evaluating the impacts of these features are ongoing and will guide improvements for the next generation MRFL. This paper describes the first study to evaluate peripheral awareness and learner effect of the MRFL prototype compared to a traditional laparoscope in a surgical simulation environment, while isolating the impact of the autotracking feature. The paper will describe the methods, outcomes, and limitations of this study and discuss future directions to isolate the impacts of multiple fields of view in a simulated surgical environment.

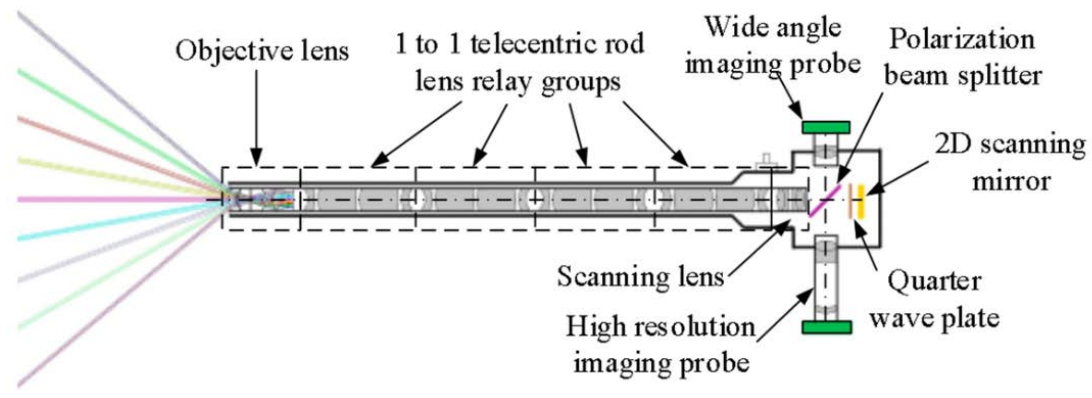

Figure 1: A schematic optical layout of the MRFL design.

\section{METHODS}

\subsection{Study Setting}

This randomized study was completed to assess learning curve and peripheral awareness when using the MRFL in comparison to a traditional laparoscope. Data was collected on both novice subjects $(\mathrm{n}=13)$ and experienced subjects $(\mathrm{n}=8)$. Novice subjects were medical and pre-medical students with no clinical experience in laparoscopy. Experienced subjects were defined as faculty and residents with a minimum of two years of clinical experience using laparoscopy. The study was completed in an isolated room at the Arizona Simulation and Technology Education Center (ASTEC). 


\subsection{Protocol}

For this study, a Fundamentals of Laparoscopic Surgery (FLS) peg transfer task was modified. Six triangles and two, six-pegged pegboards were 3D printed. The pegboards were reduced in size by one quarter, and the triangles were reduced to half the original task size. This reduced size provided additional space within the box trainer to maneuver instruments and place obstacles. One pegboard was taped around the periphery of the pegs to create a well dish. The pegboard and well dish were secured within the box trainer as shown in Figure 2. In the modified task, six numbered triangles were randomly placed on the pegboard within an FLS training box. Subjects were assigned three numbered triangles to locate and transfer to a well dish using a single Maryland grasper. If a triangle was dropped, a new triangle was assigned. In order to evaluate peripheral awareness during this task, touch sensitive obstacles were placed strategically around the pegboard and well dish. The obstacles and the trainer box base were designed to record the number and time of collisions with any metal object. The obstacles and box trainer base were painted black with metallic paint to reduce internal reflections and maintain touch sensitivity. Data from collisions were automatically recorded in the Graphic User Interface (GUI), and the number of dropped triangles was entered manually. Subjects were asked to complete this modified task using three settings: a traditional Storz laparoscope, a multi-resolution foveated laparoscope (MRFL) with autotracking (AT), and an MRFL without autotracking (NoAT). The traditional laparoscope was mobile, using the non-dominant hand of the subject to position the laparoscope. The MRFL AT was fixed in place, and the zoomed focus automatically followed the tip of the Maryland grasper. The MRFL NoAT was fixed in place allowing the subject to guide the zoomed focus using their non-dominant hand on a keyboard. The order of settings was randomly assigned to each subject to diminish learner bias. The task was repeated five times with each of the three settings, for a total of fifteen trials per subject. Before the study began, subjects were oriented to the task, Maryland grasper, and the laparoscopes with a tutorial video.

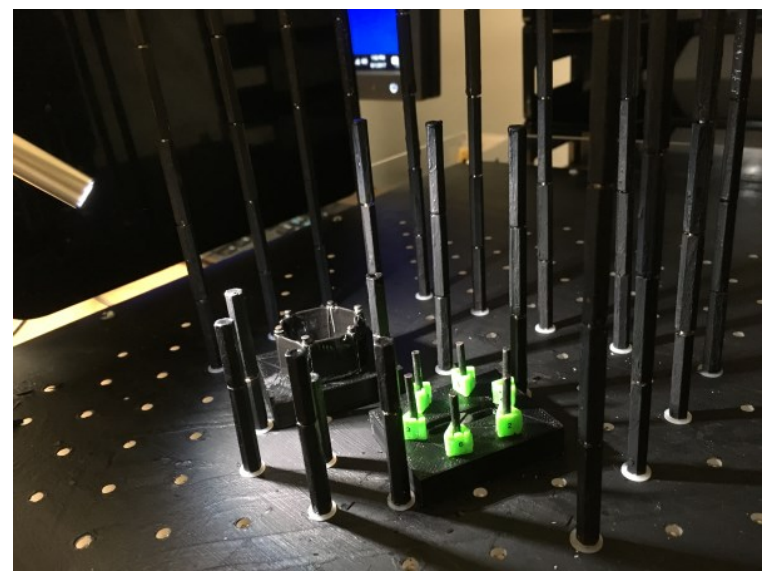

Figure 2: A picture of the modified FLS task.

\subsection{Statistical Analysis}

Data from each MRFL setting (AT and NoAT) were compared to the traditional laparoscope using a twotailed, paired Student's t-test with significance set at 95\%. The effect of autotracking was isolated by comparing the MRFL AT and MRFL NoAT data using a two-tailed, paired Student's t-test with significance set at $95 \%$. The learner effect was evaluated by plotting both the average task completion time over trial number and average number of collisions over trial number. This graph was fit with logarithmic trendlines for each setting. This statistical analysis was used separately for novice and for expert data. 


\section{RESULTS}

\subsection{Novice Data}

The novice group $(\mathrm{n}=13)$ had lower task completion times in the traditional laparoscope as compared to the MRFL AT $(p<0.001)$ and compared to the MRFL NoAT $(p<0.001)$. There was no significant difference in overall task completion time between either MRFL setting. Within each trial, task completion time for the MRFL NoAT was lower than the MRFL AT in trials two $(p<0.025)$ and five $(p<0.05)$. The learner effect with novice task completion time is shown in Figure 3. The traditional laparoscope (blue) had a trendline equation of $\mathrm{y}=-14.87 \ln (\mathrm{x})+74.111$ and $\mathrm{R}^{2}=0.8866$. The MRFL AT (red) had a trendline equation of $\mathrm{y}=-$ $26.44 \ln (x)+115.15$ and $R^{2}=0.7447$. The MRFL NoAT (black) had a trendline equation of $y=-$ $32.06 \ln (\mathrm{x})+112.53$ and $\mathrm{R}^{2}=0.7989$.

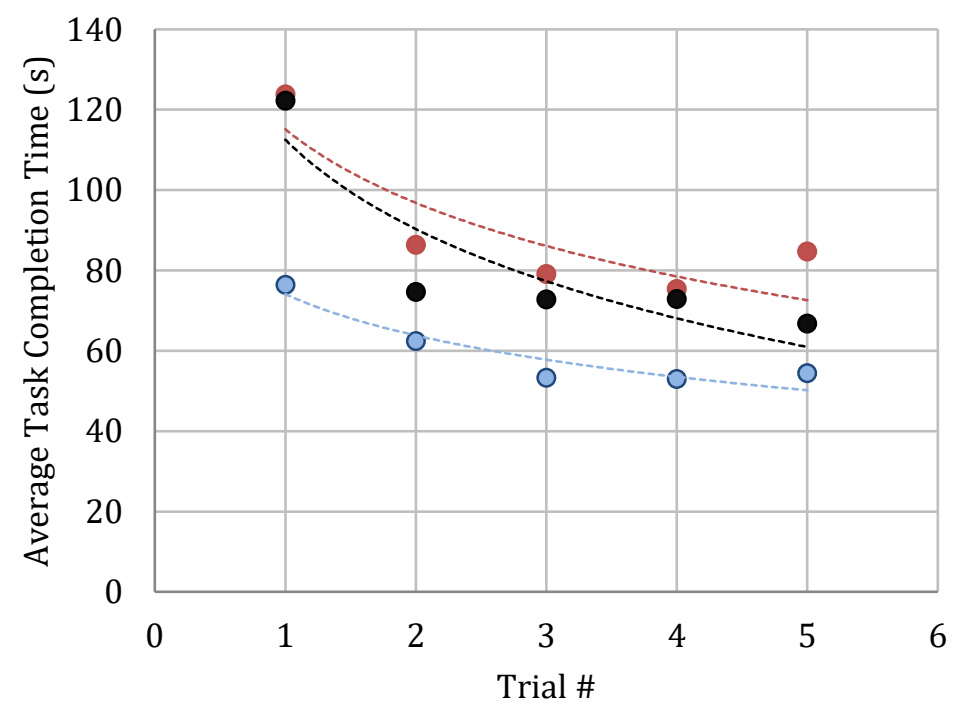

○ Traditional

- MRFL - Autotracking

- MRFL - No Autotracking Logarítmica (Traditional)

Figure 3: Novice learner effect with average task completion time across five trials.

There were no significant differences in average number of triangle drops among the traditional laparoscope $(0.11 \pm 0.36)$, MRFL AT $(0.11 \pm 0.36)$, and MRFL NoAT (0.09 \pm 0.29$)$. Additionally, no significant differences were recorded among the total time of collisions between the traditional laparoscope (9699.81 \pm 32645.10$)$, MRFL AT (11057.74 \pm 26256.51$)$, and MRFL NoAT (15358.88 \pm 49686.45$)$.

The traditional laparoscope had fewer total number of collisions than the MRFL AT $(p<0.001)$ and the MRFL NoAT $(p<0.001)$. There was no significant difference in the total number of collisions between the MRFL AT and MRFL NoAT in all five trials. The learner effect with novice total number of collisions is shown in Figure 4. The traditional laparoscope (blue) had a trendline equation of $y=-1.015 \ln (x)+6.7716$ and $\mathrm{R}^{2}=0.0991$. The MRFL AT (red) had a trendline equation of $\mathrm{y}=1.7302 \ln (\mathrm{x})+13.805$ and $\mathrm{R}^{2}=0.0323$. The MRFL NoAT (black) had a trendline equation of $y=-8.187 \ln (x)+24.239$ and $R^{2}=0.44$. 


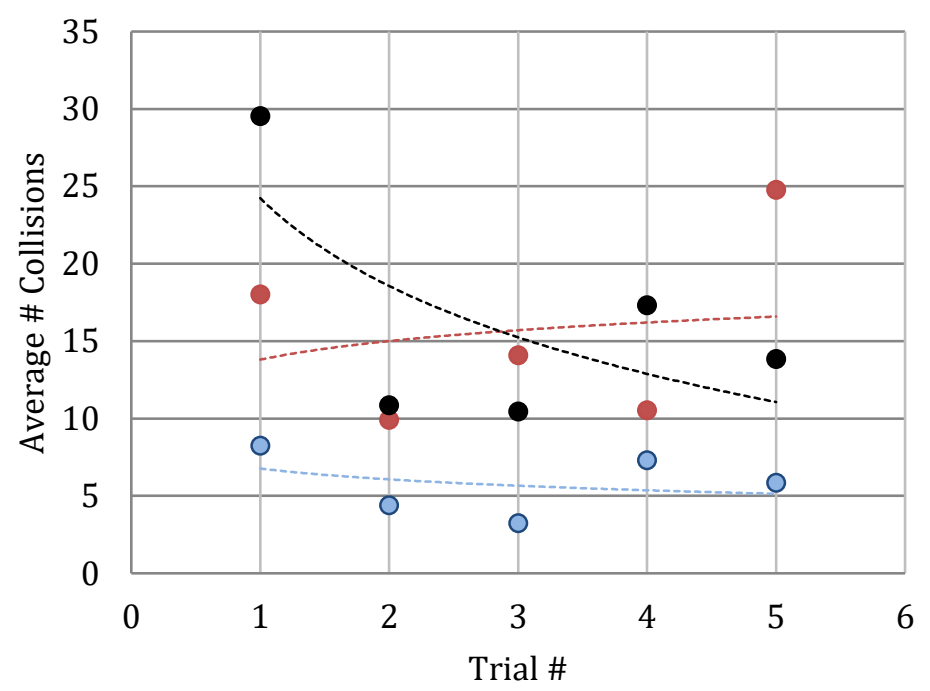

$$
\begin{aligned}
& \text { Traditional } \\
& \text { - MRFL - Autotracking } \\
& \text { - MRFL - No Autotracking } \\
& \\
& \text { Logarítmica (Traditional) } \\
& \text { Autotracking) }
\end{aligned}
$$

Figure 4: Novice learner effect with average number of collisions across five trials.

\subsection{Expert Data}

In the expert group $(\mathrm{n}=8)$, task completion times using the traditional laparoscope were lower than the MRFL AT $(p<0.001)$ and the MRFL NoAT $(p<0.001)$. The overall task completion times using the MRFL NoAT were also lower than the MRFL AT $(p<0.025)$. Task completion time for the MRFL NoAT was lower than the MRFL AT in trial one $(\mathrm{p}<0.025)$ and trial five $(\mathrm{p}<0.01)$. The learner effect with expert task completion time is shown in Figure 5. The traditional laparoscope (blue) had a trendline equation of $y=-$ $7.447 \ln (\mathrm{x})+40.406$ and $\mathrm{R}^{2}=0.8878$. The MRFL AT (red) had a trendline equation of $\mathrm{y}=-24.07 \ln (\mathrm{x})+87.499$ and $\mathrm{R}^{2}=0.837$. The MRFL NoAT (black) had a trendline equation of $\mathrm{y}=-13.45 \ln (\mathrm{x})+68.037$ and $\mathrm{R}^{2}=0.7365$.

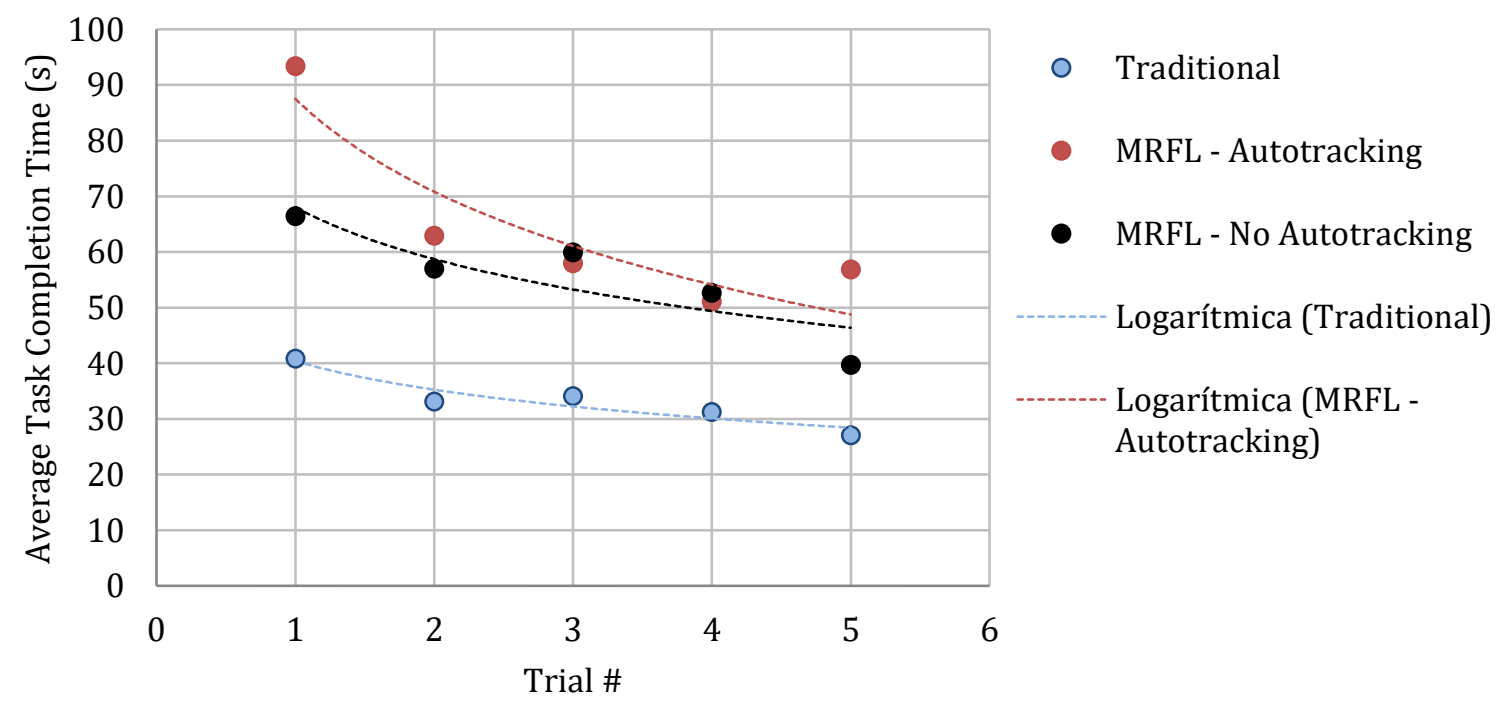

Figure 5: Expert learner effect with task completion time across five trials.

There were no significant differences in number of drops among the traditional laparoscope $(0.25 \pm 0.49)$, MRFL AT (0.18 \pm 0.45$)$, and MRFL NoAT $(0.15 \pm 0.43)$. Additionally, no significant differences were 
recorded among the total time of collisions with the traditional laparoscope (8539.22 \pm 18556.99$),$ MRFL AT (11971.14 \pm 30330.07$)$, and MRFL NoAT (11386.93 \pm 23103.47$)$.

The traditional laparoscope had fewer total number of collisions than the MRFL AT $(\mathrm{p}<0.001)$ and the MRFL NoAT $(p<0.001)$. Overall, there was no significant difference in the total number of collisions between the MRFL AT and MRFL NoAT. By trial five, the MRFL NoAT demonstrated fewer collisions than the MRFL AT $(p<0.05)$ and no significant difference from the traditional laparoscope. The learner effect with expert total number of collisions is shown in Figure 6. The traditional laparoscope (blue) had a trendline equation of $y=0.4207 \ln (x)+4.4472$ and $\mathrm{R}^{2}=0.0461$. The MRFL AT (red) had a trendline equation of $y=0.4073 \ln (x)+13.16$ and $R^{2}=0.0021$. The MRFL NoAT (green) had a trendline equation of $y=-$ $4.457 \ln (\mathrm{x})+15.093$ and $\mathrm{R}^{2}=0.8462$.

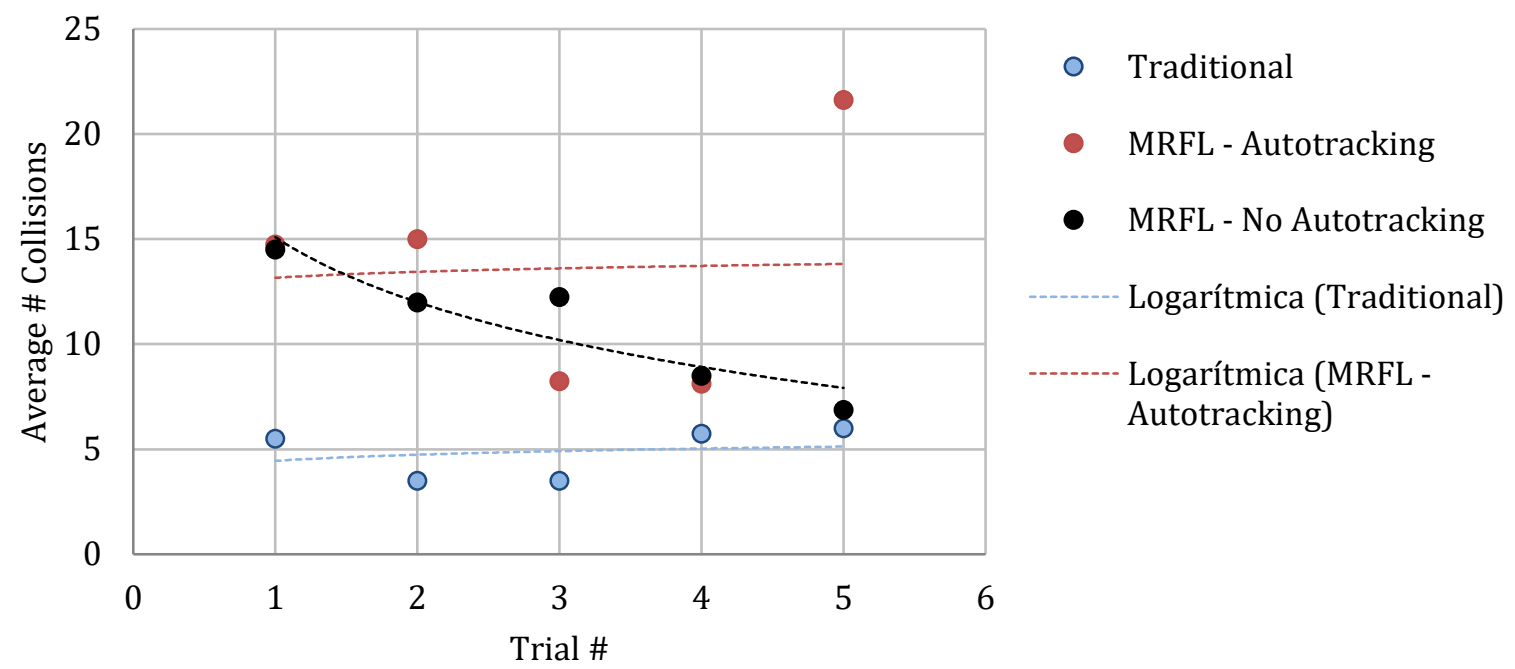

Figure 6: Expert learner effect for average number of collisions across five trials.

\section{DISCUSSION}

\subsection{Implications of the Data}

The learner effect with average task completion time in the MRFL settings was steeper in both expert and novice groups than the traditional laparoscope. This steep learner effect was indicative of subjects adjusting to this new, developing technology. In the expert group, the MRFL NoAT outcompeted the MRFL AT in total task completion time, trending toward the performance level of the traditional laparoscope. This trend justifies the continued development of the MRFL NoAT setting. At this time, the traditional laparoscope outperformed the MRFL AT and MRFL NoAT in total task completion time regardless of experience level.

In both novice and expert groups, there were no significant differences in number of triangle drops when using the traditional laparoscope, MRFL AT, or MRFL NoAT. Additional drops force the subject to find and place a new triangle, which would add time and collisions to trial data. As there were no significant differences in the number of drops across settings, this variable was effectively controlled for with this study design.

In both the novice and expert groups, there were no significant differences in total collision time when using the traditional laparoscope, MRFL AT, or MRFL NoAT. Among collision events, the collision time can vary widely: in accordance with reality, our system does not provide feedback to the subject that a collision has occurred, which can lead to collisions that last many minutes. This contributes to the large standard deviations in this data. Although total collision time is an indicator of peripheral awareness, valuable 
comparisons of these laparoscopes cannot be made with such total collision time data due to the standard deviations. Future studies may benefit from categorizing collision types by duration. The learner effect data with average number of collisions indicated a steeper learning curve in MRFL NoAT for both expert and novice groups. By trial five for the expert group, the average number of collisions for MRFL NoAT had no significant difference from the traditional laparoscope. Total number of collisions is a measure of peripheral awareness, and these data shows this prototype of MRFL NoAT has already met the level of peripheral awareness of the traditional method by trial five. This suggests that the MRFL NoAT will surpass traditional laparoscopy performance following ongoing optimization. Further improvement may be attributed to the other unique features offered by MRFL: wider field of view, increased optimal working distance, and multiple views displayed simultaneously.

In comparison to the MRFL AT and MRFL NoAT at all experience levels, the traditional laparoscope demonstrated superior peripheral awareness, shorter task completion times, and flatter learning curves. In the experienced group, the MRFL NoAT trended toward the same peripheral awareness and task completion times as the traditional laparoscope. Given the limitations of the MRFL, the MRFL NoAT shows the most promise to improve peripheral awareness and task performance in future studies.

\subsection{Limitations}

There were multiple limitations identified within this study. The learner effect data demonstrated that a performance plateau had not been reached by the MRFL AT and MRFL NoAT by trial five. This learning curve adds significant variation to data points and averages collected in our MRFL trials. Ideally, data collection would begin at the learner effect plateau to better compare peripheral awareness when using the traditional laparoscope and MRFL. This method would double the experimental time and would not be conducive to recruiting subjects, especially in the expert pool. By including novice subjects, we can better identify the impact of these learning curve variations.

Throughout this study, limitations to the autotracking technology were also identified. First, the autotracking is programmed to follow the tip of the instrument. In this study, the instrument tip is distinguished by the direction it enters the surgical field, light reflection off the metal tip, and by motion in front of a stationary background. In laparoscopic surgery, the direction the instruments enter the field will change and multiple moving instruments are often used simultaneously. The autotracking technology with the current tracking programming could not account for these standard surgical variations. Additionally, all subjects verbally noted that the autotracking was too sensitive to small instrument movements. This high sensitivity may have played a role in the increased experimental times and number of collisions seen in the MRFL AT group.

There was a distinct difference in visual clarity between the traditional laparoscope and MRFL, further complicated by decreased light capturing by the MRFL. The maximum light source level with the MRFL produced a darker image than the traditional laparoscope at half maximum light. This difference made it difficult to read the numbers on the triangles using the zoomed perspective of the MRFL, while the traditional laparoscope could more easily read the numbers even without moving. The impact of this visual quality difference on the study cannot be isolated from these data. This difference may be associated with the internal fiber optics within the traditional laparoscope, while the MRFL uses internal lenses. Updating the internal optics and light capturing systems within the MRFL should eliminate visual quality differences, which may cut down on collisions and completion times by reducing the need for extraneous movement. As the MRFL is a developing technology, the use of fiber optics is cost prohibitive in early prototypes. Using an optical filter to control for visual clarity differences may be a more cost effective option in future development. 


\subsection{Future Directions}

This study provided valuable information to guide the continued development of MRFL and the experimental design of upcoming studies. In subsequent versions of MRFL, adjustments should be made to the shape and size of the MRFL optical housing, the amount of light entering the MRFL, the visual quality within the zoomed view, and the sensitivity of the autotracking. The optical housing in this version of the MRFL had bulky projections in all directions. This bulky design limited the options for experimental setup and optimal range of motion for the instrument. A housing design that is more linear would address this issue. Additionally, the amount of light entering the MRFL probe was notably less than the light entering the traditional laparoscope. To enhance the visual clarity of the zoomed image, adjustments should be made to increase the amount of light captured by the MRFL. The overall visual clarity of the MRFL zoomed image could be improved with fiber optic technology to match the visual quality of traditional laparoscope. In respect to autotracking, the technology needs to be optimized to decrease the sensitivity of the zoomed view tracking to small instrument movements. Additionally, the autotracking program should be modified to limit tracking to the area of interest when multiple moving objects are within the surgical view. Although the benefits of the autotracking technology were not demonstrated in this study, the above modifications may address some of these challenges to using the MRFL AT.

In upcoming studies, focus should be directed toward isolating the effect of multiple fields of view and controlling for differences in visual quality, as compared to traditional laparoscopy. Multiple fields of view provided by the MRFL have potential to limit the amount of movement and overall time needed to insert and remove tools in the periphery, while maintaining focus on a central area of interest. Motion tracking of the instruments and eye tracking may provide additional insights into the impacts of multiple fields of view. Additionally, visual quality differences need to be controlled for in upcoming studies by matching the optics within the traditional laparoscope or by including a visual filter over the traditional laparoscope as an additional experimental group.

\section{REFERENCES}

Hata, K., Kazama, S., Kawai, K., Kiyomatsu, T., Tanaka, J., Tanaka, T., Nishikawa, T., Yamaguichi, H., Ishihara, S., Sunami E., Kitayama, J., and Watanabe, T. 2014. "Laparoscopic surgery for ulcerative colitis: a review of the literature". Surgery Today vol 45, pp 933-938.

Hua, H., and Liu, S. 2008. "Dual-sensor foveated imaging system". Applied Optics vol 47, pp 317.

Jaschinski, T., Mosch, C.G., Eikermann, M., Neugebauer, E.A., and Sauerland, S. 2018. "Laparoscopic versus open surgery for suspected appendicitis". Cochrane Database of Systemic Reviews issue 11, Art. No.: CD001546.

Qin, Y., and Hua, H. 2016. "Continuously zoom imaging probe for the multi-resolution foveated laparoscope". Biomedical Optics Express vol 7, pp 1175-1182.

Qin, Y., and Hua, H. 2016. "Optical design and system engineering of a multiresolution foveated laparoscope". Applied Optics vol 55, pp. 3058-3068.

Qin, Y., Hua, H., and Nguyen, M. 2013. "Multiresolution foveated laparoscope with high resolvability". Optics Letters vol 38, pp 2191-2193.

Qin, Y., Hua, H., and Nguyen, M. 2014. "Characterization and in-vivo evaluation of a multiresolution foveated laparoscope for minimally invasive surgery". Biomedical Optics Express vol 5, pp. 2548-2562.

Wu, K.L., Lee, K.C., Liu, C.C., Chen H.H., and Lu C.C. 2017. "Laparoscopic versus Open Surgery for Diverticulitis: A Systematic Review and Meta-Analysis". Digestive Surgery, Vol. 34, pp. 203-215. 
AUTHOR BIOGRAPHIES

MARISSA LOVETT is a Medical Student at the University of Arizona College of Medicine - Tucson and a Research Specialist at the Arizona Simulation Technology and Education Center (ASTEC). She holds an MS in Physiology from the University of Arizona. Her research interests include optimization of telesimulation training, evaluation of optical medical technology, and design and testing of augmented reality applications to enhance medical education. Her email address is Lovett1@email.arizona.edu.

JEREMY KATZ is a PhD student at the University of Arizona's College of Optical Sciences. He holds a BS in Optical Engineering from the University of Arizona. His research interests include lens design, optical engineering, and endoscopic systems, focusing on applications in surgical imaging. His email address is JJikatz@email.arizona.edu.

SANGYOON LEE is an Assistant Research Professor of College of Optical Sciences at University of Arizona. He holds a PhD in Computer Science and Engineering from Pohang University of Science and Technology (POSTECH), South Korea. His research interests lie in virtual and augmented realities, especially in depth perception and user interfaces. His email address is sylee@optics.arizona.edu.

DAVID BIFFAR is the Director of Operations for the Arizona Simulation Technology and Education Center (ASTEC) and the Center for Advanced Technologies in Healthcare (CATH). He is a Certified Healthcare Simulation Operations Specialist (CHSOS) and an Accreditation Reviewer for the Society for Simulation in Healthcare. His research interests include human factors and usability testing in healthcare environments, interprofessional education modalities, and virtual reality exposure therapy for generalized anxiety disorders. His email address is dbiffar@surgery.arizona.edu.

MIKE NGUYEN, MD, MPH is an Associate Professor of Urology at the Keck School of Medicine of USC. He conducts research in several areas including the application of novel surgical cameras for minimally invasive surgery, prostate cancer prevention, and behavioral approaches to the prevention of kidney stone disease and other benign urologic conditions. His email address is mike.nguyen@med.usc.edu.

HONG HUA, Fellow of SPIE and OSA, is a Professor of Optical Sciences in the College of Optical Sciences at the University of Arizona. She holds a Ph.D. in Optical Engineering from Beijing Institute of Technology. Dr. Hua's current research focuses on optical technologies enabling advanced 3D displays, and microscopic and endoscopic imaging systems for medicine. Her email address is hhua@optics.arizona.edu.

ALLAN HAMILTON, MD, FACS is the Executive Director for the Arizona Simulation Technology and Education Center (ASTEC) and a Professor of Neurosurgery at the University of Arizona College of Medicine - Tucson. His current research interests include interprofessional education modalities and virtual patient simulation. His email address is allan@surgery.arizona.edu. 